\title{
LncRNA ACTA2-AS1 suppress colon adenocarcinoma progression by sponging miR-4428 upregulation BCL2L11
}

Qingyun Pan ${ }^{1+}$, Ying Huang ${ }^{3+}$, Yirui Wang ${ }^{2+}$, Deke $\mathrm{Li}^{4+}$ and Changjiang Lei ${ }^{5^{*}}$ (I)

\begin{abstract}
Background: Long non-coding RNA is considered to be essential to modulate the development and progression of human malignant cancers. And long non-coding RNA can act as crucial modulators by sponging the corresponding microRNA in tumorigenesis. We aimed to elucidate the function of ACTA2-AS1 and its molecular mechanism in colon adenocarcinoma.

Materials and methods: The expression of ACTA2-AS1, miR-4428 and BCL2L11 in colon adenocarcinoma tissues were detected via qRT-PCR. SW480 and HT29 cells were transfected with shRNA ACTA2-AS1, OE ACTA2-AS1, miRNA mimics of miR-4428, miR-4428 inhibitor, si-BCL2L11 and over-expression of Si-BCL2L11. Cell proliferation, colony formation and apoptosis were respectively assessed using CCK-8 assay, colony assay and flow cytometry. Luciferase reporter assay was performed to verify the targets of ACTA2-AS1 and miR-4428. Tumor subcutaneous xenograft mode was constructed to explore tumor growth in vivo.
\end{abstract}

Results: ACTA2-AS1 was obviously downregulated in human colon adenocarcinoma tissues and colon adenocarcinoma cell lines. Silence or over-expression of ACTA2-AS1 promoted or inhibited cell proliferation and colony formation abilities, and regulated apoptosis. The silence of ACTA2-AS1 resulted in the decrease of Bax and increase of Bal2, while restored in OE ACTA2-AS1 group when compared with the control transfected cells. In addition, luciferase reporter assay revealed that ACTA2-AS1 interacted with miR-4428 and suppressed its expression. miR-4428 could bind to 3' untranslated region of BCL2L11 and modulated the expression of BCL2L11 negatively. Knockdown of ACTA2-AS1 and over-expression of BCL2L11 reversed the biological function that ACTA2-AS1 mediated by knockdown ACTA2-AS1 alone.

Conclusion: Our data demonstrated that ACTA2-AS1 could suppress colon adenocarcinoma progression via sponging miR-4428 to regulate $B C L 2 L 11$ expression.

Keywords: Colon adenocarcinoma, ACTA2-AS1, miR-4428, BCL2L11

*Correspondence: leicj_biology@163.com

${ }^{\dagger}$ Qingyun Pan, Ying Huang, Yirui Wang, Deke Li are co-first authors

${ }^{5}$ Department of General Surgery, The Fifth Hospital of Wuhan, Wuhan 430000, Hubei, People's Republic of China

Full list of author information is available at the end of the article

\begin{abstract}
Introduction
Colorectal cancer $(\mathrm{CRC})$ is one of the most frequently diagnosed tumors with poor prognosis and the most common CRC is colon adenocarcinoma (COAD) [1]. The development and progress of COAD is a multistep process in which accumulating genetic changes can play an important role. Although great progression has been made in surgery, chemotherapy, radiotherapy, and targeted drugs, there is no actual achievements in the
\end{abstract}

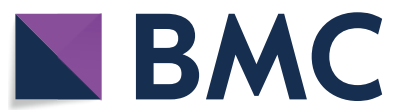

(c) The Author(s) 2021. This article is licensed under a Creative Commons Attribution 4.0 International License, which permits use, sharing, adaptation, distribution and reproduction in any medium or format, as long as you give appropriate credit to the original author(s) and the source, provide a link to the Creative Commons licence, and indicate if changes were made. The images or other third party material in this article are included in the article's Creative Commons licence, unless indicated otherwise in a credit line to the material. If material is not included in the article's Creative Commons licence and your intended use is not permitted by statutory regulation or exceeds the permitted use, you will need to obtain permission directly from the copyright holder. To view a copy of this licence, visit http://creativeco mmons.org/licenses/by/4.0/. The Creative Commons Public Domain Dedication waiver (http://creativecommons.org/publicdomain/ zero/1.0/) applies to the data made available in this article, unless otherwise stated in a credit line to the data. 
overall survival rate of COAD patients [2]. Hence, the investigation of promising therapeutic targets and molecular mechanism involved in the carcinogenesis of COAD remains especially crucial for the early diagnosis, timely treatment, and prognosis.

It is known that long non-coding RNAs (lncRNAs) are RNA molecules with more than 200 nucleotides [3, 4]. Generally, IncRNAs modulate the expression level of targeted genes at the post-transcriptional period [5]. More and more lncRNAs have been found to involve in many aspects of cellular homeostasis, such as angiogenesis, metastasis, cell proliferation, immunity adjustment, genomic stability, and so on [6,7]. Previous studies also suggest that lncRNAs are involved in the tumorigenesis through multiple mechanisms, such as transcriptional regulation, protein post-translational regulation miRNA regulation and so on [8]. Previous studies also proved that a growing number of lncRNAs play crucial roles in COAD tumorigenesis and development, such as ZDHHC8P1, FOXD3-AS1, and ZEB1-AS [9-11]. Therefore, lncRNAs could be regarded as potential diagnostic and prognostic biomarkers for human cancers.

LncRNA ACTA2-AS1 (ACTA2 Antisense RNA 1) is located at 10q23.31 with five exons [12]. Recent studies revealed that dysregulation of ACTA2-AS1 has been found to be closely related to poor prognosis of several cancers, such as cervical cancer, hepatocellular carcinoma, liver cancer, breast cancer and lung adenocarcinoma [13-15]. According to previous studies, ACTA2-AS1 may play an important role involved in the development of human cancers. However, the role of ACTA2-AS1 in COAD and its underlying molecular mechanisms remains unclear. Our previous studies found an obvious decrease of ACTA2-AS1 expression in both COAD cell lines and COAD tissues, and we presumed that ACTA2-AS1 may act as a crucial regulator in COAD. Therefore, this study is aimed to explore the specific function of ACTA2-AS1 in COAD and the molecular mechanisms involved.

\section{Materials and methods}

\section{Cell lines and COAD tissues}

Normal human colon mucosal epithelial cell line (CCD18Co) and six COAD cell lines (SW480, HT29, LS174T, HCT116 and DLD-1) were procured from ATCC. The above cells were cultured in RPMI 1640 medium or DMEM (Invitrogen, USA) with 10\% fetal bovine serum (Invitrogen, USA) and $1 \%$ Penicillin/Streptomycin (Sigma-Aldrich, USA) at $37{ }^{\circ} \mathrm{C}$ incubator containing 5\% $\mathrm{CO}_{2} .82$ newly diagnosed patients with COAD in The Fifth Hospital of Wuhan were included in the present study. All the experiments were carried out according to the principles of The Fifth Hospital of Wuhan.

\section{Cell transfection}

The design and construction of shRNAs and si-RNA for ACTA2-AS1 and BCL2L11, the synthesis of pcDNA 3.1, miR-4428 mimics vector, and the construction of a lentiviral vector overexpressing ACTA2-AS1 and BCL2L11 were separately conducted by Genechem (Shanghai, China). The transfection was carried out using Lipofectamine 2000 reagent (Invitrogen, USA) according to the guideline of manufacturer. The sequences of genes were as followed:

\section{si-ACTA2-AS1\#1: 5'-UAGAUUAUUAUGUCUUCC CAG-3' \\ Si-ACTA2-AS1\#2: 5'-UAGUAAAGCAACAUUCUU GGA-3' \\ si-BCL2L11: 5'-UUAAAUAACGUGAACAUGCUG-3' miR-4428 mimics: 5'-GUUCCUCUGCCCUUGUAC CUCG-3'}

\section{RNA extract and quantitative real-time PCR (qRT-PCR) assay}

Trizol reagent (TaKaRa, China) was used to extract total RNA according to the manufacturer's instructions. The PrimeScript RT Master Mix (TaKaRa, China) was used to reverse-transcribe lncRNA and mRNA. The SYBR Premix Ex Taq II Kit (TaKaRa, China) was employed to carry out Real-time PCR. The relative RNA expression was normalized to the expression levels of U6 and GAPDH. The primers used for quantitative PCR were as follows:

ACTA2-AS1:

5'-GTGGTTCTGGTTTGCCTGAT-3' (forward), 5'-CTGGCCCTGTAACACCAGAT-3' (reverse); miR-4428:

5'-GTTCCTCTGCCCTTGTACCTCG-3' (forward), 5'-GCGCGCGTA ACAGTCTACAGC-3' (reverse); $B C L 2 L 11$ :

5'-TAAGTTCTGAGTGTGACCGAGA-3' (forward), 5'-GCTCTGTCTGTAGGGAGGTAGG-3' (reverse); U6:

5'-CTCGCTTCGGCAGCACA-3' (forward), 5'-AACGCTTCACGAATTTGCGT-3' (reverse); GAPDH:

5'-TGCACCACCAACTGCTTAGC-3' (forward), 5'-GGCATGCACTGTGGTCATGAG-3' (reverse).

\section{Cytoplasm and nuclear localization}

The NE-PER ${ }^{\text {TM }}$ Cytoplasmic and Nuclear Extraction Reagents Kit (Thermo Fisher Scientific) was done to confirm the cytoplasmic localization of ACTA2-AS1 in COAD cells. Following the manufacturer's instructions, the COAD cells nuclear and cytoplasmic constituents were sorted and collected. Afterward, qRT-PCR was used to evaluate the ACTA2-AS1 expression in the nucleus 
and cell cytoplasm, respectively. GAPDH was used as the cytoplasm localization control while U6 was for the nucleus localization control.

\section{Cell viability and colony assay}

The Cell Counting Kit-8 (CCK-8) (Dojindo, Japan) experiment was used to measure cell viability. COAD cells were seeded into 96-well plates at $37{ }^{\circ} \mathrm{C}$ incubator with $5 \% \mathrm{CO}_{2}$. After transfection, the OD 450 values were measured at $0,24,48$, and $72 \mathrm{~h}(\mathrm{~h})$ via CCK- 8 assay after incubation for $2 \mathrm{~h}$ at $37{ }^{\circ} \mathrm{C}$. Then, the absorbance values were measured on a microplate reader at $450 \mathrm{~nm}$. For colony formation assay, 2000 SW480 and HT29 cells were seeded in 6-well plates and cultured for seven to ten days at $37^{\circ} \mathrm{C}$ incubator with $5 \% \mathrm{CO}_{2}$. Then, the cells were washed twice with PBS (phosphate-buffered saline), fixed with $4 \%$ paraformaldehyde (Sinopharm Chemical, China) and stained with crystal violet (Sigma, USA) for $15 \mathrm{~min}$ respectively. The clone spots were counted observed under a microscope (Olympus, Japan) with 5 random view fields.

\section{Apoptotic assay}

Apoptotic assay was performed using V-FITC Annexin and PI Apoptosis Detection Kit (Beyotime, China). SW480 and HT29 were fixed in 70\% cooled ethanol and stained with Annexin V-FITC and PI for $20 \mathrm{~min}$ at room temperature according to the protocol, and then cell apoptosis was detected by flow cytometer.

\section{Dual luciferase reporter assay}

Through starbase 2.0 , we found the miR-4428 binding site in ACTA2-AS1 and the downstream target gene of miR-4428 is BCL2L11. The $3^{\prime}-$ UTR of BCL2L11 and ACTA2-AS1 containing wild type (wt) and mutant type (mut) reporter vectors were purchased from Beijing TransGen Biotech Co., (Beijing, China). miR-4428 mimics binding sequence was inserted downstream of the firefly luciferase gene in psi-CHECK2 vector to synthesis the BCL2L11-wt or ACTA2-AS1-wt and psi-CHECK2BCL2L11-mut or ACTA2-AS1-mut plasmids, respectively. The wt and mut plasmids subsequently were co-transfected into in SW480 and HT29 cells cells with negative control and miR-4428 mimics. After transfection for $48 \mathrm{~h}$, the cells were lysed and the relative luciferase activity was measured via the Dual-luciferase reporter Assay System (Promega, Madison, WI, USA).

\section{RNA pull-down assay}

For RNA pull-down assay, the streptavidin-coated magnetic beads (Life Technologies, CA, USA) were covered by biotinylated ACTA2-AS1 (Bio-ACTA2-AS1) and BioOligo according to its instruction and transfected into
$1 \times 10^{6} \mathrm{SW} 480$ and HT29 cells at $50 \mathrm{nM}$ as a final concentration for $48 \mathrm{~h}$. Subsequently, $0.7 \mathrm{~mL}$ lysis buffer $(5 \mathrm{mM}$ $\mathrm{MgClz}, 100 \mathrm{mM} \mathrm{KCl}, 20 \mathrm{mM}$ Tris (pH 7.5), 0.3\% NP-40) and complete protease inhibitor cocktail (Roche Applied Science, IN) were added into the cell pellets, then the cell lysates were incubated together with the RNA-tagged beads for the co-immunoprecipitation (Invitrogen, Carlsbad, CA, USA). The RNA-RNA complexes were subsequently collected by centrifugation at $10,000 \mathrm{r}$ for $10 \mathrm{~min}$ and then the miR-4428 enrichment level was detected with qRT-PCR analysis.

\section{Western blotting}

Protein was extracted using Radioimmunoprecipitation assay (RIPA, Beyotime, China). Protein samples were separated through $10 \%$ sodium dodecyl sulfate-polyacrylamide gel electrophoresis (SDS-PAGE) and then transferred onto PVDF (polyvinylidene fluoride) membranes (Thermo Fisher Scientific, USA). Membranes were blocked in 5\% bovine serum albumin (BSA) for $2 \mathrm{~h}$ and then incubated with primary antibodies, including anti-Bcl-2, anti- Bax and GAPDH (Cell Signaling Technology, USA) overnight at $4{ }^{\circ} \mathrm{C}$. Next day, after washed with PBST for three times, the membranes were incubated with the HRP-conjugated secondary antibodies (Cell Signaling Technology, USA) at $37{ }^{\circ} \mathrm{C}$ for $1 \mathrm{~h}$. Finally, the intensity of the bands was visualized by using enhanced chemiluminescence (ECL).

\section{Tumor xenografts in nude mice}

BALB/c nude mice (16-20 g, 5 weeks of age) were purchased from Shanghai Animal Laboratory Center (Shanghai, China). Cells transfected with sh-NC or sh-ACTA2-AS1 were digested with $0.25 \%$ trypsin, diluted in PBS, counted by trypan blue staining, adjusted to a concentration of $1.0 \times 10^{\wedge} 7$ cells $/ \mathrm{mL}$, and $0.1 \mathrm{~mL}$ $\left(1.0 \times 10^{\wedge} 6\right.$ cells $)$ of this solution was injected hypodermically into the back flank of each mice. Tumor size was calculated every 7 days. Additionally, the present investigation was approved by the ethics committee of The Fifth Hospital of Wuhan.

\section{Bioinformatics analysis and binding sites prediction}

The Cancer Genome Atlas (TCGA, https://www.curel ine.com/the-cancer-genome-atlas.html) database from Gene Expression Profiling Interactive Analysis (GEPIA, http://gepia.cancer-pku.cn/) was used to explore the expression of ACTA2-AS1 in COAD patients. The potential miR-4428 binding sites to 3UTR of BCL2L11 was predicted by Starbase (http://starbase.sysu.edu.cn/) and the ACTA2-AS1 binding sites to the miR-4428 was predicted by lncBASE database (http://carolina.imis.athen ainnovation.gr/diana_tools/web/index.php? $r=\operatorname{lncbasev} 2 /$ 


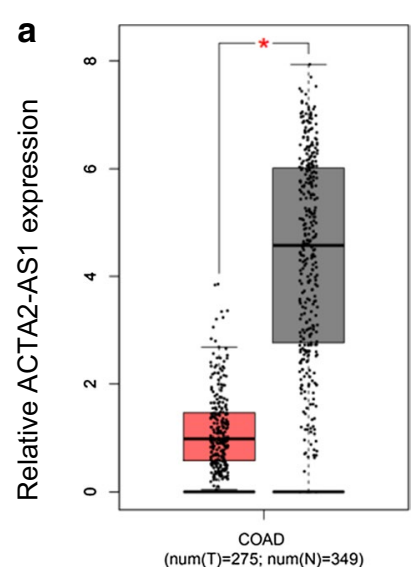

b

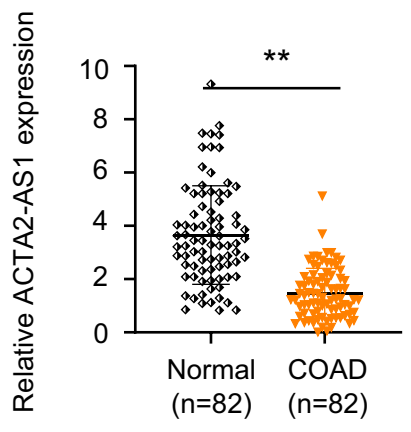

c

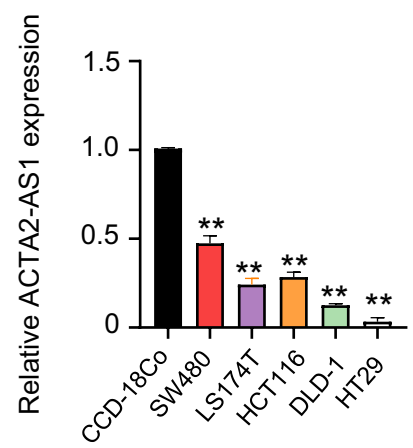

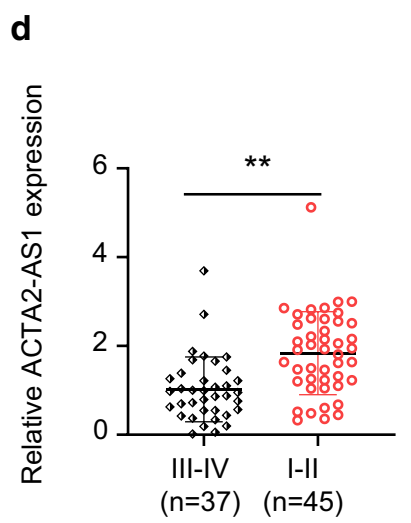

e

f
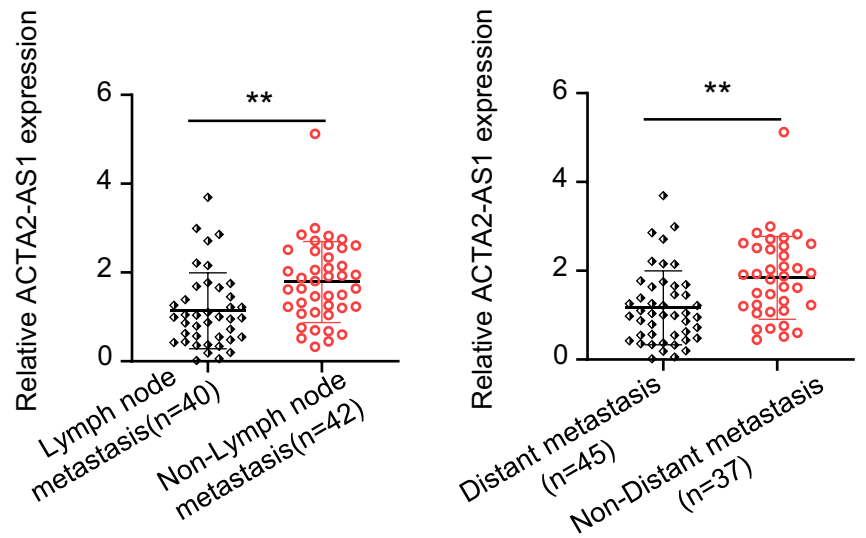

g

h

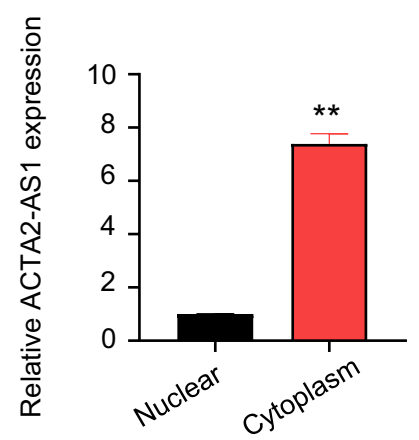

Fig. 1 LnCRNA ACTA2-AS1 was down-expression in COAD tissue samples and cells. a ACTA2-AS1 was down-expressed in COAD samples from TCGA database. b Relative ACTA2-AS1 expression in 82 pairs of COAD tissue samples and their adjacent normal tissues using qRT-PCR assay. $\mathbf{c}$ Relative ACTA2-AS1 expression in different COAD cell lines (SW480, HT29, LS174T, HCT116 and DLD-1) and normal human colon mucosal epithelial cell line CCD-18Co. $\mathbf{d}$ Relative ACTA2-AS1 expression in different clinical stages of COAD. e Downregulation of ACTA2-AS1 in COAD was associated with lymph node metastasis. $\mathbf{f}$ Downregulation of ACTA2-AS1 in COAD was associated with distant metastasis. $\mathbf{g}$ The KM-Plotter was used to evaluate survival in COAD patients. Patients with low ACTA2-AS1 expression had worse overall survival than those with high ACTA2-AS1 expression. $\mathbf{h}$ Localization of ACTA2-AS1 in COAD cells using nuclear cytoplasm separation experiment. ${ }^{*} \mathrm{p}<0.05 ;{ }^{* *} \mathrm{p}<0.01$

index-predicted) to study the potential crossing network among ACTA2-AS1, miR-4428 and BCL2L11.

\section{Statistical analysis}

The data in in study were presented as mean \pm standard deviation (SD). The data were visualized through the GraphPad Prism 7.0 software. All statistical analysis was 
conducted via SPSS 20.0. $\mathrm{p}<0.05$ presented statistically significant.

\section{Results}

LnCRNA ACTA2-AS1 was down-regulated in COAD tissue samples and cells

To investigate the role of lncRNA ACTA2-AS1 in COAD tissue samples, we firstly downloaded data from TCGA database and exhibited that the expression of ACTA2AS1 was obviously upregulated in normal samples compared with COAD samples (Fig. 1a). Then, 82 pairs of human COAD tissue samples and their corresponding adjacent non-tumor samples were detected by qRTPCR. As shown as Fig. 1b, the expression of ACTA2AS1 was remarkably low in COAD tissues. In addition, this study detected the expression level of ACTA2-AS1 in six COAD cell lines (SW480, HT29, LS174T, HCT116 and DLD-1) and normal human colon mucosal epithelial cell line CCD-18Co, finding that ACTA2-AS1 expression was extremely lower in COAD cells than in CCD18Co cell (Fig. 1c). ACTA2-AS1 expression was detected in different clinical stages of COAD, suggesting that the expression of ACTA2-AS1 was negatively correlated with advanced TNM stage. We also observed that ACTA2AS1 expression was further significantly down-regulated in COAD patients that had lymph node or distal metastasis compared with those without metastasis (Fig. 1e and f). Furthermore, the median expression value of

Table 1 Relationship between ACTA2-AS1 expression and their clinicopathologic parameters in COAD tissue samples

\begin{tabular}{llll}
\hline Variable & \multicolumn{2}{l}{ ACTA2-AS1 expression } & P value \\
\cline { 2 - 3 } & Low $(\mathrm{n}=41)$ & High $(\mathrm{n}=41)$ & \\
\hline Age & & & \\
$<55$ & 21 & 23 & 0.507 \\
$\geq 55$ & 20 & & \\
Gender & & 26 & 0.651 \\
Male & 24 & 15 & \\
Female & 17 & & $0.002^{*}$ \\
Distant metastasis & & 24 & \\
Negative & 10 & 17 & 0.184 \\
Positive & 31 & & \\
Family history & & 25 & \\
Yes & 19 & 16 & $0.001^{*}$ \\
No & 22 & & \\
TNM stage & & 31 & \\
I/II & 15 & 10 & \\
III/IV & 26 & 32 & \\
Lymph node metastasis & 17 & 9 & \\
Negative & 24 & & \\
Positive & & & \\
\hline
\end{tabular}

ACTA2-AS1 in COAD tissues was the cut-off value and then divided the tissues into high expression group $(\mathrm{n}=41)$ and low expression group $(\mathrm{n}=41)$, KaplanMeier survival analysis suggested that high-ACTA2-AS1 expression was associated with longer survival time in patients with COAD (Fig. 1g). Next, we further demonstrated that ACTA2-AS1 was mainly localized in cytoplasm (Fig. 1h). These results revealed that prominent low-expression of ACTA2-AS1 was detected in COAD samples and low expression of ACTA2-AS1 was related to poor prognosis in patients with COAD (Table 1).

\section{Effect of ACTA2-AS1 on the proliferation and apoptosis of COAD cells}

In order to examine the effect of ACTA2-AS1 in COAD, we silenced ACTA2-AS1 expression in SW480 using shRNAs and over-expressed ACTA2-AS1 in HT29 cell lines, respectively. The knockdown and over-expression efficiency results demonstrated that ACTA2-AS1 expression was significantly decreased or increased by sh-ACTA2-AS1 or OE ACTA2-AS1 transfection, respectively (Fig. 2a), suggesting that the effect of ACTA2-AS1 was inhibited or promoted. The results of CCK- 8 assay disclosed that knockdown of ACTA2-AS1 obviously increased the proliferative ability of SW480 cells in contrast to the sh-NC groups, while cell proliferation was obviously inhibited in the OE ACTA2-AS1 group compared with vector group (Fig. 2b). Similarly, the clone formation assay revealed that inhibition of ACTA2-AS1 increased the number of colony formation in SW480 cells lines compared with the sh-NC group, while the ability of colony formation was inhibited in the OE ACTA2-AS1 group (Fig. 2c). Next, the apoptosis role of ACTA2-AS1 in COAD cell was detected via flow cytometric analysis assay, indicating that the cell apoptosis rate was obviously decreased or increased after ACTA2-AS1 down-regulation or up-regulation (Fig. 2d). Taken together, lncRNA ACTA2-AS1 could inhibit COAD cell proliferation, and meanwhile promote apoptosis.

\section{LncRNA ACTA2-AS1 sponged miR-4428 in COAD cells}

To verify the potential molecular target of ACTA2-AS1 involved in COAD cells, an online bioinformatics tool lncBASE was detected. As shown as Fig. 3a, there was a potential complementary sequence between ACTA2-AS1 and miR-4428. Luciferase reporter assay was assessed to detect and verify the interaction of ACTA2-AS1 and miR-4428, finding that the relative luciferase activities were reduced in ACTA2-AS1-wt transfected cells while that in ACTA2-AS1-mut groups displayed no change (Fig. 3b). Moreover, RNA pull-down assay also exhibited that miR-4428 were enriched in bio-ACTA2-AS1 group (Fig. 3c). Transfection of sh-ACTA2-AS1 markedly 


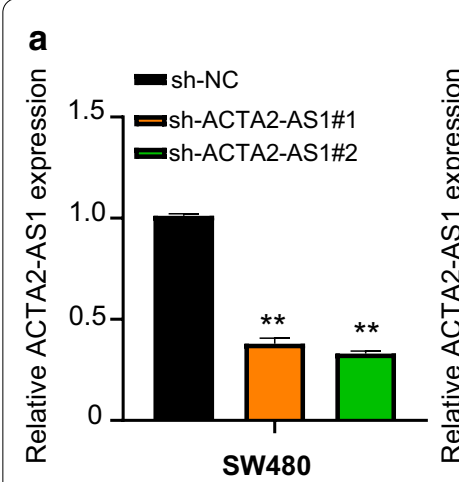

C
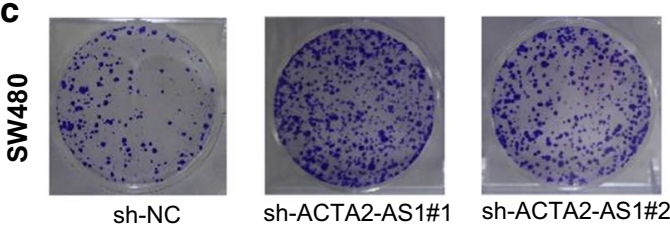

- sh-NC

चsh-ACTA2-AS1\#1

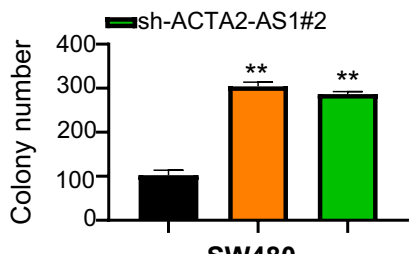

SW480

d
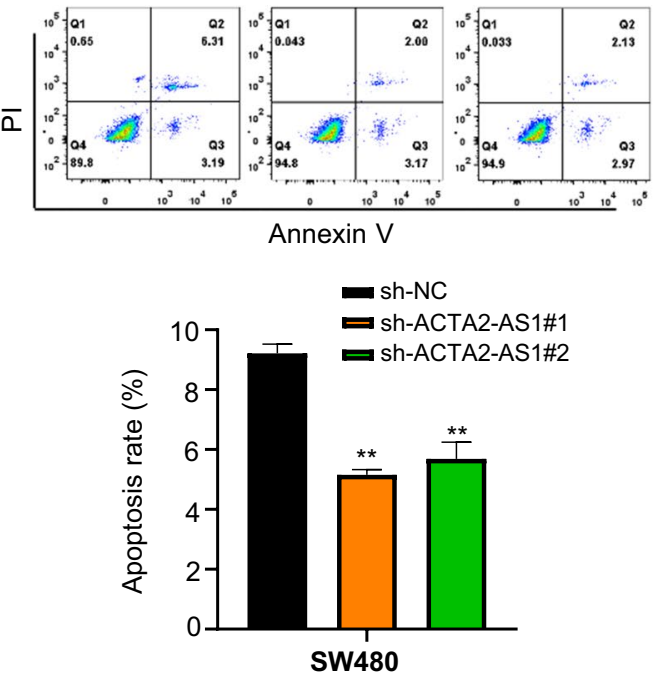

b
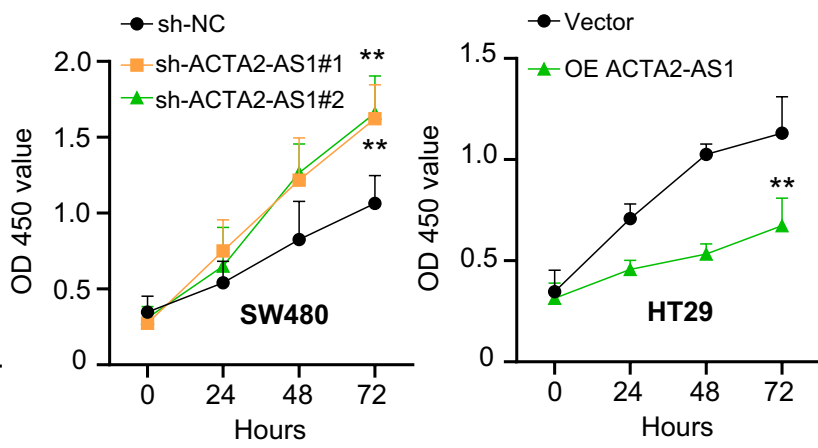

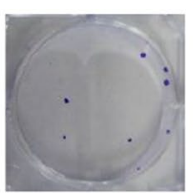

OE ACTA2-AS1

- Vector

- OE ACTA2-AS1

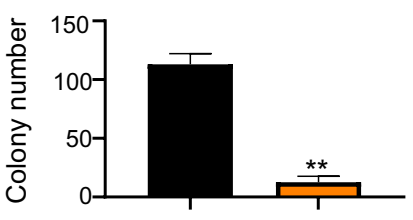

HT29

$\bar{\alpha}$
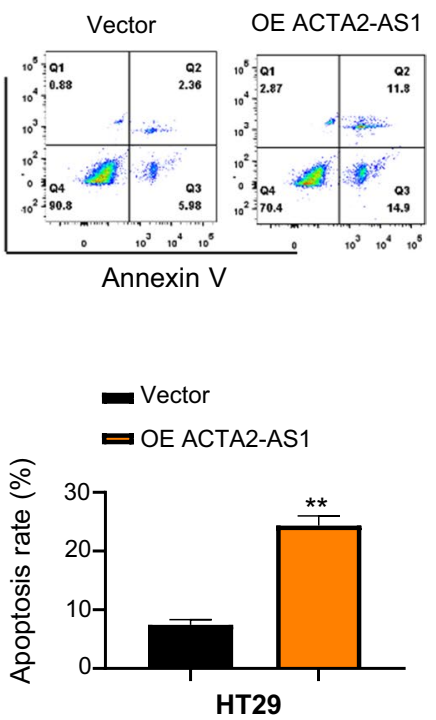

Fig. 2 Effect of ACTA2-AS1 on the proliferation and apoptosis of COAD cells. a qRT-PCR analysis of knockdown and overexpression efficiency of ACTA2-AS1 in SW480 and HT29 cells transfected with sh-NC, sh-ACTA2-AS1, empty vector and OE ACTA2-AS1 respectively. b CCK-8 assay results of cell viability in SW480 and HT29 cells transfected with sh-NC, sh-ACTA2-AS1, empty vector and OE ACTA2-AS1 respectively. c Colony formation in SW480 and HT29 cells transfected with sh-NC, sh-ACTA2-AS1, empty vector and OE ACTA2-AS1. d Flow cytometric analysis showing the effects of sh-ACTA2-AS1 and OE ACTA2-AS1 on the cell apoptosis in SW480 and HT29 cells. $p<0.05$; ${ }^{* *} p<0.01$ 
increased the expression of miR-4428, while transfection of OE ACTA2-AS1 decreased the expression of miR-4428 (Fig. 3d). In addition, qRT-PCR assay also disclosed that miR-4428 was increased in 82 pairs of human COAD tissue comparing with adjacent non-tumor tissues (Fig. 3e). Meanwhile, a negative relationship was observed between the expression of ACTA2-AS1 and miR-4428 in COAD tissues (Fig. 3f). The above results confirmed that ACTA2-AS1 could serve as a sponge of miR-4428 in COAD cells.

\section{miR-4428 directly interacted with BCL2L11}

To predict the downstream direct target mRNA of miR4428, an online bioinformatics tool Starbase was carried out. The results showed that an underlying miR-4428 binding sites was detected in the 3'-untranslated region

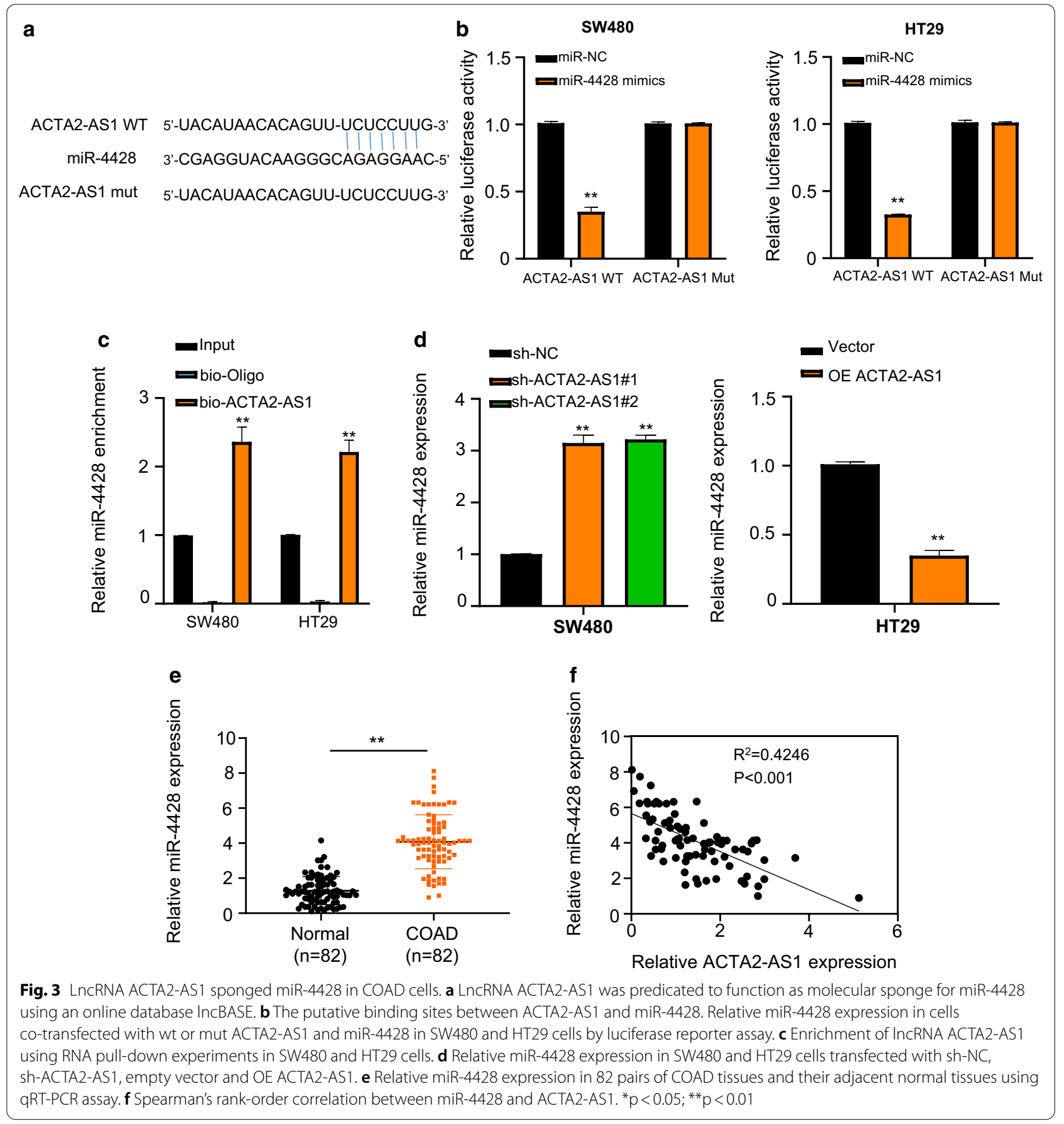




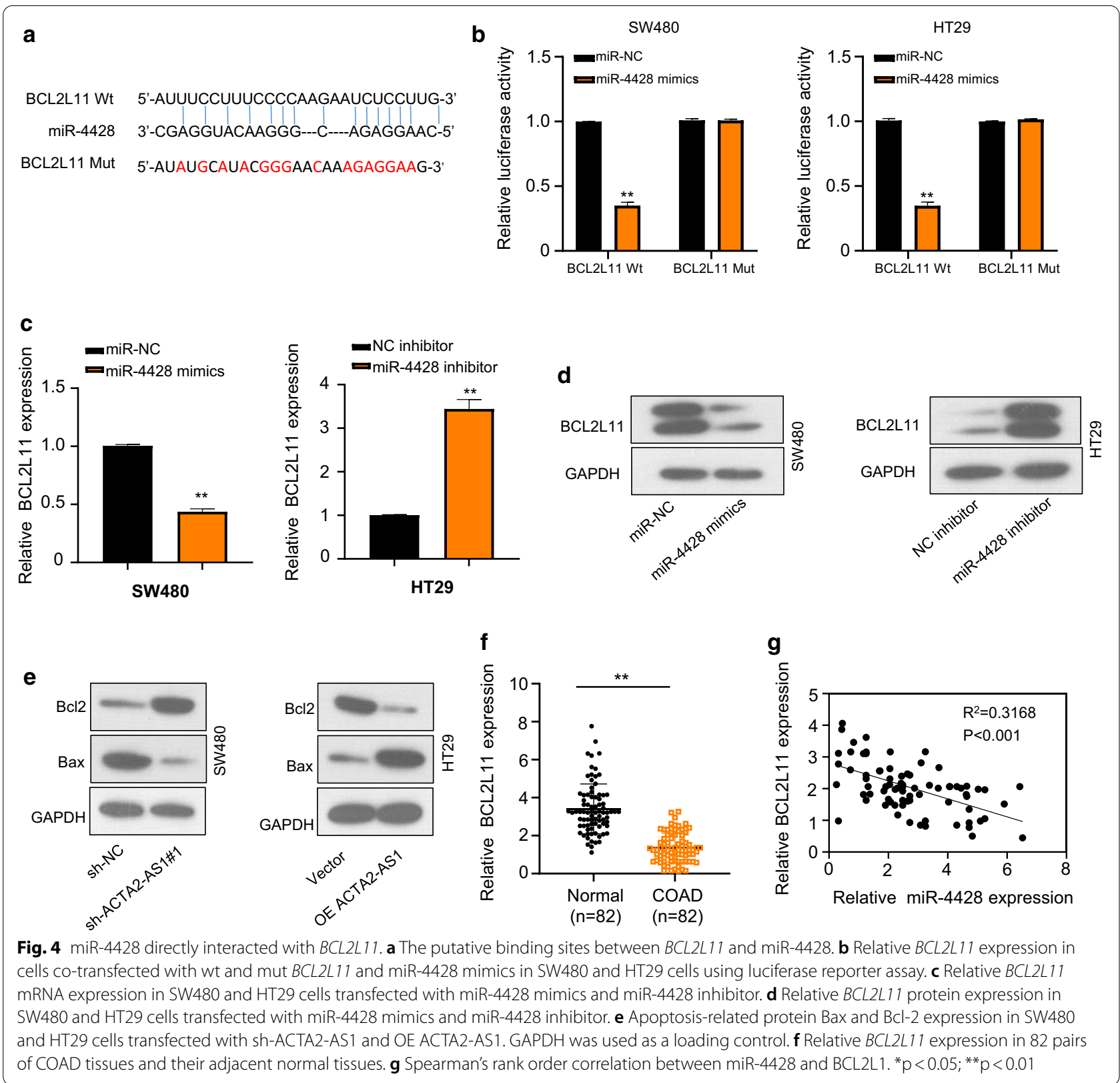

(3UTR) of BCL2L11 (Fig. 4a). Then, luciferase reporter assay verified that the relative luciferase activities were reduced in $B C L 2 L 11$-wt transfected cells while no significant change of relative luciferase activity was measured in the BCL2L11-mut group (Fig. 4b). Data from qRTPCR and Western blot demonstrated that both mRNA and protein expression level of BCL2L11 decreased or increased in COAD cell lines transfected with miR4428 mimics or miR-4428 inhibitor, respectively (Fig. 4c and $\mathrm{d}$ ). The proteins of apoptosis markers $\mathrm{Bcl}-2$ and Bax were assessed by Western blotting, suggesting that $\mathrm{Bcl}-2$ were up-regulated in the sh-ACTA2-AS1 group in comparison with the control group. However, the level of Bax was suppressed. Next, OE ACTA2-AS1 was transfected into HT29 cells, the expression trend of the above proteins was reversed (Fig. 4e). Conversely, the downregulation of ACTA2-AS1 resulted in the decrease of Bax when compared with the control transfected cells, while increased in OE ACTA2-AS1 group in comparison with the vector group (Fig. 4e). Next, we identified that $B C L 2 L 11$ was memorably down-regulated in 82 pairs of human COAD tissue compared to that in matched adjacent normal tissue (Fig. 4f). And the expression of $B C L 2 L 11$ was negatively related miR-4428 expression in 
human COAD tissues via Spearman's rank-order analysis (Fig. 4g). These results illustrated that BCL2L11 directly interacts with miR-4428.

\section{LncRNA ACTA2-AS1 suppressed COAD progression by sponging miR-4428 upregulation BCL2L11}

MRNA expression of $B C L 2 L 11$ was reduced in HT29 cells by transfection with si-BCL2L11 (Fig. 5a), and its mRNA and protein expression could be rescued in SW480 cells by sh-ACTA2-AS1 co-transfection with the miR-4428 inhibitor and BCL2L11, while the expression of $B C L 2 L 11$ was increased by transfection with OE ACTA2AS1, and its expression could be suppressed in the cells by co-transfection with the miR-4428 mimics and siBCL2L11 in HT29 (Fig. 5b-d). Furthermore, both cell proliferation and colony formation were increased in the cells transfected of sh-ACTA2-AS1 in comparison with sh-NC group, while restored in sh-ACTA2-AS1 + miR4428 inhibitor and sh-ACTA2-AS1 + BCL2L11 group. Correspondingly, cells transfected with OE ACTA2-AS1, both cell proliferation and colony formation were inhibited compared with sh-NC group and increased by cotransfection with OE ACTA2-AS1 and miR-4428 mimics or OE ACTA2-AS1 and over-expression of BCL2L11 (Fig. 5e-g). In addition, flow cytometry assays manifested that silence of miR- 4428 or over-expression of BCL2L11 could reverse the promoting apoptosis effect of ACTA2AS1 (Fig. 5h). These results indicated that ACTA2-AS1 suppressed COAD progression by sponging miR-4428 upregulation BCL2L11.

\section{The suppressive roles of LncRNA ACTA2-AS1 in COAD in vivo experiments}

Then, in order to evaluate the effects of ACTA2-AS1 in vivo experiments, the $B A L B / c$ nude mice were employed to construct tumor subcutaneous xenografts, the results suggested that the volume and weight of the xenograft tumors were larger or smaller in the sh-ACTA2-AS1 group or in OE ACTA2-AS1 group comparing the sh-NC and vector group respectively (Fig. 6a and $\mathrm{b}$ ). The proteins of apoptosis markers Bcl-2 and Bax were also assessed in subcutaneous xenograft tumor, the expression of Bcl-2 was showed to significantly increase in sh-ACTA2-AS1 group in comparison with the sh-NC group, while decreased in OE ACTA2-AS1 group in comparison with the vector group. Conversely, the downregulation of ACTA2-AS1 resulted in the decrease of Bax when compared with the control transfected cells, while restored in OE ACTA2-AS1 group in comparison with the vector group (Fig. 6c). A schematic representation of the ACTA2-AS1 mechanism of regulation of CRC progression is presented in Fig. 7.

\section{Discussion}

It is widely acknowledged that lncRNA could competitively sponge and regulate the expressions of miRNA to regulate tumorigenesis [16-18]. For instance, lncRNAROR promotes tumor growth and metastasis of colon cancer cell by targeting miR-145 [19]. In addition, MNX1-AS1 and ELFN1-AS1 were found to facilitate cell proliferation through regulating miR-218-5p/SEC61A1 axis in colon adenocarcinoma [20,21]. Recent evidence indicated that high-expression of ACTA2-AS1 was positively associated with poor prognosis in COAD patients, accelerating the pathological activities via regulating miR-4644/TRIM44 [22]. LINC00342 and LINC00491 could accelerate progression of COAD by regulating miR-545-5p/MDM2 axis and sponging miR-145 respectively $[23,24]$.

Recently, it was reported that ACTA2-AS1 is significantly over-expressed in cervical cancer, upregulating SMAD3 expression by competitively sponging miR143-3p [15]. However, ACTA2-AS1 may act as a tumor suppressor in lung adenocarcinoma and liver cancer

\footnotetext{
(See figure on next page.)

Fig. 5 LnCRNA ACTA2-AS1 suppressed COAD progression by sponging miR-4428 upregulation BCL2L11. a Relative BCL2L11 mRNA expression in HT29 cells transfected with sh-NC and sh- ACTA2-AS1. b Relative BCL2L11 mRNA expression in SW480 transfected with sh-NC, sh-ACTA2-AS1, sh-ACTA2-AS1 + miR-4428 inhibitor and sh-ACTA2-AS1 + BCL2L11. And relative BCL2L11 mRNA expression in HT29 cells transfected with OE ACTA2-AS1, OE ACTA2-AS1 + miR-4428 mimics, and OE ACTA2-AS1 + si-BCL2L11, respectively. c Relative BCL2L11 mRNA protein in SW480 transfected with sh-NC, sh-ACTA2-AS1, sh-ACTA2-AS1 + miR-4428 inhibitor and sh-ACTA2-AS1 + BCL2L11. And relative BCL2L11 protein expression in HT29 cells transfected with OE ACTA2-AS1, OE ACTA2-AS1 + miR-4428 mimics, and OE ACTA2-AS1 + si-BCL2L11, respectively. d Apoptosis-related protein Bax and Bcl-2 expression in SW480 and HT29 cells transfected with sh- ACTA2-AS1, sh-ACTA2-AS1 + miR-4428 inhibitor and sh-ACTA2-AS1 + BCL2L11, and OE ACTA2-AS1, OE ACTA2-AS1 + miR-4428 mimics, and OE ACTA2-AS1 + si-BCL2L11, respectively. e CCK-8 assay of cell viability in SW480 and HT29 cells transfected with sh- ACTA2-AS1, sh-ACTA2-AS1 + miR-4428 inhibitor and sh-ACTA2-AS1 + BCL2L11, and OE ACTA2-AS1, OE ACTA2-AS1 + miR-4428 mimics, and OE ACTA2-AS1 + si-BCL2L11. f Colony formation in SW480 and HT29 cells transfected with sh- ACTA2-AS1, sh-ACTA2-AS1 + miR-4428 inhibitor and sh-ACTA2-AS1 + BCL2L11, and OE ACTA2-AS1, OE ACTA2-AS1 + miR-4428 mimics, and OE ACTA2-AS1 + si-BCL2L11. g Flow cytometric analysis showing the effects of the cell apoptosis in SW480 and HT29 cells transfected with sh-ACTA2-AS1, sh-ACTA2-AS1 + miR-4428 inhibitor and sh-ACTA2-AS1 + BCL2L11, and OE ACTA2-AS1, OE ACTA2-AS1 + miR-4428 mimics, and OE ACTA2-AS1 + si-BCL2L11, respectively. ${ }^{* *} \mathrm{p}<0.01$
} 


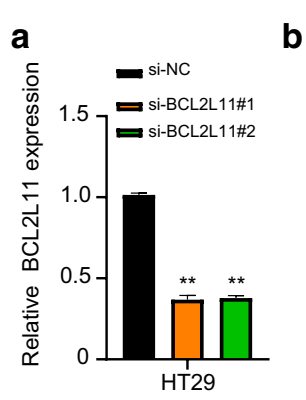

b
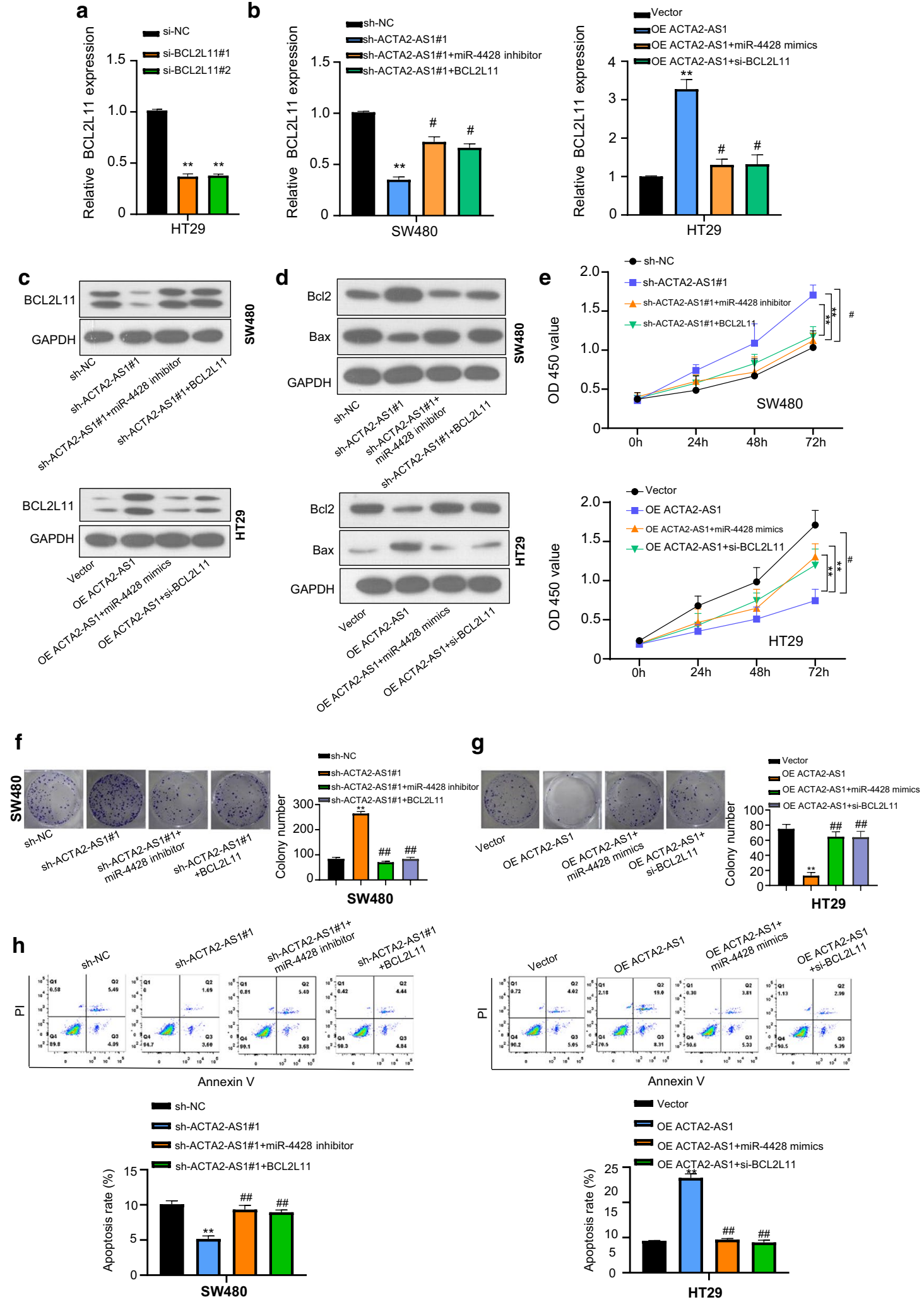

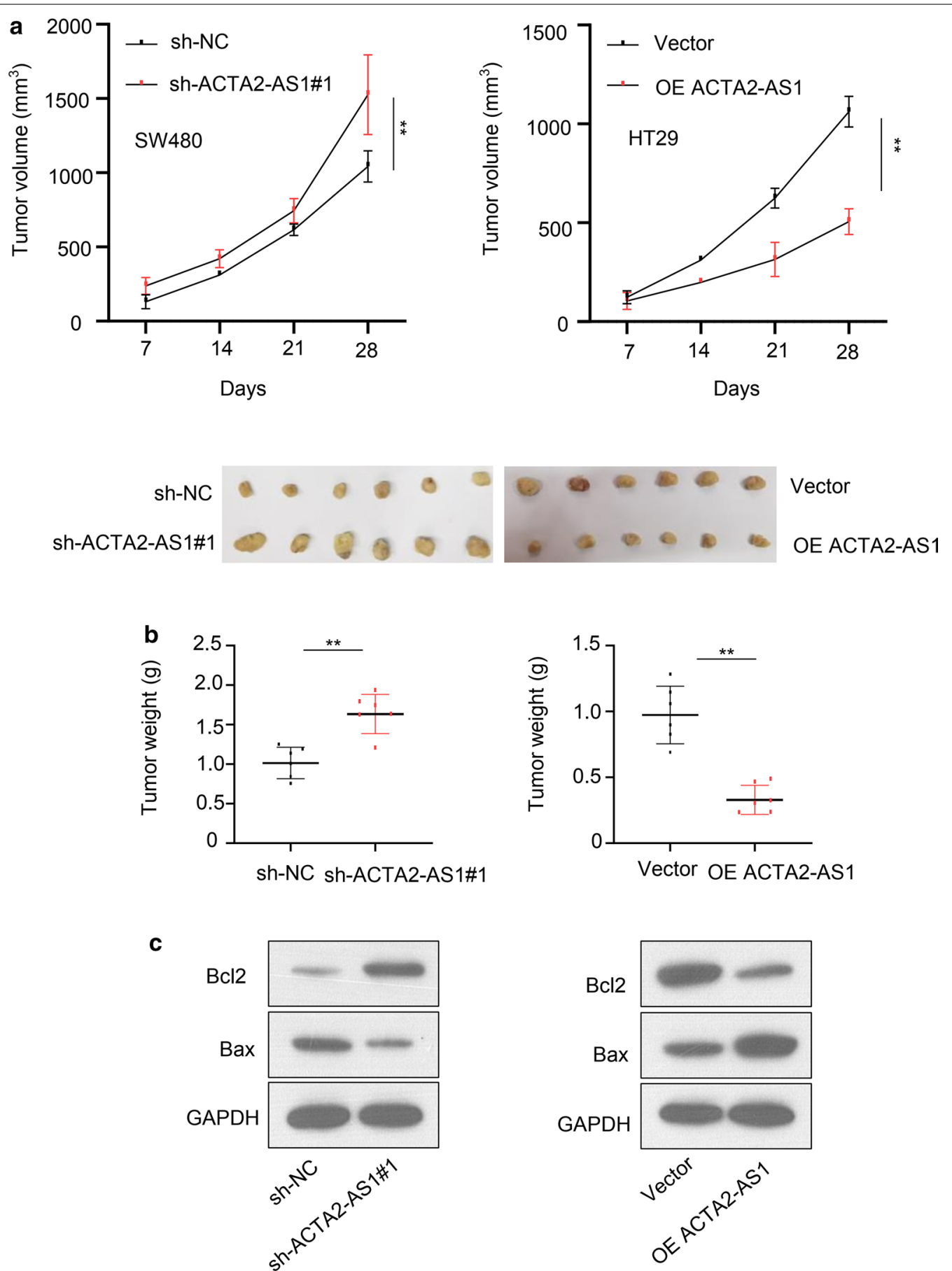

Fig. 6 The suppressive roles of LnCRNA ACTA2-AS1 in COAD in vivo. a Tumor volume and tumor diagram in nude mice implanted subcutaneously with SW480 and HT29 cells transfected with sh-ACTA2-AS1 and IncRNA ACTA2-AS1 OE respectively. b Tumor weight in nude mice implanted subcutaneously with SW480 and HT29 cells transfected with sh-ACTA2-AS1 and IncRNA ACTA2-AS1 OE respectively. c Apoptosis-related protein Bax and Bcl-2 expression in subcutaneous tumor transfected with sh-ACTA2-AS1 and IncRNA ACTA2-AS1 OE. GAPDH was used as a loading control. ${ }^{*} p<0.05$

cell via sequestering miR-378a-3p and miR-4428 to upregulate the expression of SOX7. However, the role of ACTA2-AS1 in COAD is not completely researched yet. This study suggested that low-expression of ACTA2-AS1 contributing to poor prognosis of COAD through KM-plot analysis. Inhibition or overexpression 

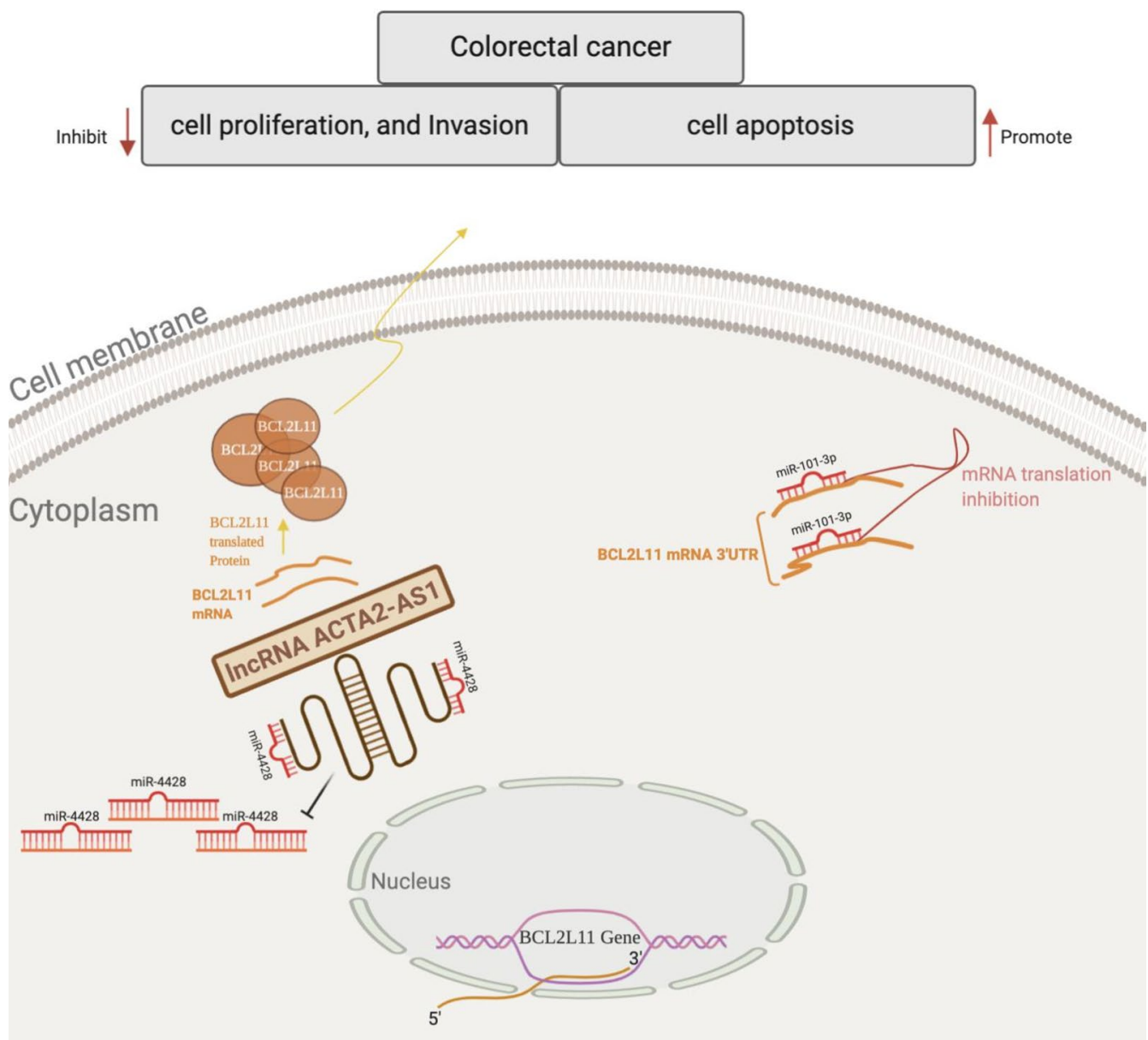

Fig. 7 A schematic representation of the ACTA2-AS1 mechanism of regulation of CRC progression

of ACTA2-AS1 promoted or inhibited cell proliferation, colony formation and induced apoptosis, demonstrating that ACTA2-AS1 might suppress the progress of COAD. Previously, Ying et al. showed that miR-4428 could bind with ACTA2-AS1 and posed positive effects on growth, migration and epithelial-mesenchymal transition process in non-small cell lung cancer [14]. Evidences from our study indicated that miR-4428 could sponge ACTA2-AS1 and regulated the expression of $B C L 2 L 11$ negatively. Rescue assays suggested that the impaired COAD cells growth and facilitated apoptosis triggered by over-expression of ACTA2-AS1 could be recovered by knockdown of $B C L 2 L 11$ or over-expression of miR-4428.

$B C L 2 L 11$ (also known as BIM) is a member of BCL-2 family, inducing apop-tosis and inhibiting autophagy by inactivating BCL2 or by activating BAX-BAK1 and by bridging BECN1 or DYNLL1, respectively [25-27]. According to previous reports, BCL2L11 is involved in biological pro-cesses in a variety of solid tumors such as ovarian cancer, endometrial adenocarcinoma, prostate tumor and gastric cancer [28-30]. Cumulating evidence has demonstrated that the dysregulation of miRNAs plays crucial roles in the pathology tumorigenesis by directly tar-geting the $3^{\prime}$-UTRs of mRNA of target genes. For example, it is reported that the expression of BCL2L11 is a direct target of miR-24 in gastric cancer, regulating cell growth and apoptosis. In human endometrial adenocarcinoma, miR-106a mimics cotransfected with wild-type $B C L 2 L 11$ 3'-UTR markedly inhibited the relative luciferase activities of RL95-2 and HEC-1B cells, suggesting that BCL2L11 is the direct target of miR-106a [28]. In this study, we found the 3'-UTRs of BCL2L11 could sponge miR-4428. Additionally, $B C L 2 L 11$ was low-expressed in COAD tissues and negatively correlated with $\mathrm{miR}-4428$, and restoration of BCL2L11 expression completely rescued the inhibitory effect of up-regulation of ACTA2-AS1 in COAD cells. These results revealed that $B C L 2 L 11$ was 
directly regulated by miR-4428 and might play a crucial role in COAD Additional file 1.

Taken together, ACTA2-AS1 plays a suppressive role in COAD via decoying miR-4428 to augment the expression of BCL2L11, inhibiting cell proliferation and promoting apoptosis. Our study demonstrated that ACTA2-AS1 may be a novel prognostic marker and therapeutic target biomarker in COAD.

\section{Supplementary information}

The online version contains supplementary material available at https://doi. org/10.1186/s12935-021-01769-3.

Additional file 1: Additional Figures.

\section{Acknowledgements}

Thanks to all the members in our department for their help in experiment methods suggestion and data collection.

\section{Authors' contributions}

$\mathrm{CL}$ designed the project, collected data, analyzed the data and drafted the manuscript. QP and YH did all the experiments, YW involved in data collection and analysis. DL revised and corrected the manuscript. All authors read and approved the final manuscript.

\section{Funding}

Not applicable.

\section{Availability of data and materials}

The data and materials have been included in this manuscript, and can contect author with email followed to cite.E-mail: leicj_biology@163.com.

\section{Ethics approval and consent to participate}

The present investigation was approved by the ethics committee of The Fifth Hospital of Wuhan. All experiments are performed after the consent of the patients or family members.

\section{Consent for publication}

We would like to submit the enclosed manuscript entitled "LncRNA ACTA2AS1 suppress colon adenocarcinoma progression by sponging miR-4428 upregulation BCL2L11", which we wish to be considered for publication in Cancer Cell International.

\section{Competing interests}

The authors report no conflicts of interest in this work.

\section{Author details}

${ }^{1}$ Department of Blood Endocrinology, The Fifth Hospital of Wuhan, Wuhan 430000, Hubei, People's Republic of China. ${ }^{2}$ Department of Oncology, The Fifth Hospital of Wuhan, Wuhan 430000, Hubei, People's Republic of China. ${ }^{3}$ Department of Pharmacy, The Fifth Hospital of Wuhan, Wuhan 430000 , Hubei, People's Republic of China. ${ }^{4}$ Department of Anesthesiology, The Fifth Hospital of Wuhan, Wuhan 430000, Hubei, People's Republic of China. ${ }^{5}$ Department of General Surgery, The Fifth Hospital of Wuhan, Wuhan 430000, Hubei, People's Republic of China.

Received: 12 November 2020 Accepted: 10 January 2021

Published online: 12 April 2021

\section{References}

1. Dekker E, Tanis PJ, Vleugels JLA, Kasi PM, Wallace MB. Colorectal cancer. Lancet. 2019;394(10207):1467-80.
2. Mobley LR, Kuo TM. Demographic disparities in late-stage diagnosis of breast and colorectal cancers across the USA.J Racial Ethn Health Disparities. 2017:4(2):201-12

3. Chi Y, Wang D, Wang J, Yu W, Yang J. Long non-coding RNA in the pathogenesis of cancers. Cells. 2019;8(9):1015.

4. Ponting CP, Oliver PL, Reik W. Evolution and functions of long noncoding RNAs. Cell. 2009;136(4):629-41.

5. Chen F, Li Z, Deng C, Yan H. Integration analysis for novel IncRNA markers predicting tumor recurrence in human colon adenocarcinoma. J Transl Med. 2019;17(1):299.

6. Kong S, Tao M, Shen X, Ju S. Translatable circRNAs and IncRNAs: driving mechanisms and functions of their translation products. Cancer Lett. 2020;483:59-65.

7. Akhade VS, Pal D, Kanduri C. Long noncoding RNA: genome organization and mechanism of action. Adv Exp Med Biol. 2017;1008:47-74.

8. Li N, Zhan X. Identification of clinical trait-related IncRNA and mRNA biomarkers with weighted gene co-expression network analysis as useful tool for personalized medicine in ovarian cancer. EPMA J. 2019;10(3):273-90.

9. Li C, Liu T, Zhang Y, Li Q, Jin LK. LnCRNA-ZDHHC8P1 promotes the progression and metastasis of colorectal cancer by targeting miR-34a. Eur Rev Med Pharmacol Sci. 2019;23:1476-86.

10. Ni X, Ding Y, Yuan H, Shao J, Yan Y, Guo R, Luan W, Xu M. Long non-coding RNA ZEB1-AS1 promotes colon adenocarcinoma malignant progression via miR-455-3p/PAK2 axis. Cell Prolif. 2020;53(1):e12723.

11. Wu Q, Shi M, Meng W, Wang Y, Hui P, Ma J. Long noncoding RNA FOXD3AS1 promotes colon adenocarcinoma progression and functions as a competing endogenous RNA to regulate SIRT1 by sponging miR135a-5p. J Cell Physiol. 2019;234(12):21889-902.

12. Polev DE, Karnaukhova IK, Krukovskaya LL, Kozlov AP. ELFN1-AS1: a novel primate gene with possible microRNA function expressed predominantly in human tumors. Biomed Res Int. 2014;2014:398097.

13. Zhou RJ, Lv HZ. Knockdown of ACTA2AS1 promotes liver cancer cell proliferation, migration and invasion. Mol Med Rep. 2019;19(3):2263-70.

14. Ying K, Wang L, Long G, Lian C, Chen Z, Lin W. ACTA2-AS1 suppresses lung adenocarcinoma progression via sequestering miR-378a-3p and miR-4428 to elevate SOX7 expression. Cell Biol Int. 2020;44(12):2438-49.

15. Luo L, Wang M, Li X, Luo C, Tan S, Yin S, Liu L, Zhu X. A novel mechanism by which ACTA2-AS1 promotes cervical cancer progression: acting as a ceRNA of miR-143-3p to regulate SMAD3 expression. Cancer Cell Int. 2020;20:372.

16. Xiong W, Qin J, Cai X, Xiong W, Liu Q, Li C, Ju Y, Wang Q, Li Y, Yang Y. Overexpression LINC01082 suppresses the proliferation, migration and invasion of colon cancer. Mol Cell Biochem. 2019;462(1-2):33-40.

17. Si Y, Yang Z, Ge Q, Yu L, Yao M, Sun X, Ren Z, Ding C. Long non-coding RNA Malat1 activated autophagy, hence promoting cell proliferation and inhibiting apoptosis by sponging miR-101 in colorectal cancer. Cell Mol Biol Lett. 2019;24:50.

18. Rinn JL, Chang HY. Genome regulation by long noncoding RNAs. Annu Rev Biochem. 2012;81:145-66.

19. Zhou P, Sun L, Liu D, Liu C, Sun L. Long Non-Coding RNA lincRNA-ROR Promotes the Progression of Colon Cancer and Holds Prognostic Value by Associating with miR-145. Pathol Oncol Res. 2016;22(4):733-40.

20. Ye Y, Gu B, Wang Y, Shen S, Huang W. E2F1-mediated MNX1-AS1-miR218-5p-SEC61A1 feedback loop contributes to the progression of colon adenocarcinoma. J Cell Biochem. 2019;120(4):6145-53.

21. Dong L, Ding C, Zheng T, Pu Y, Liu J, Zhang W, Xue F, Kang P, Ma Y. Extravehicular vesicles from human umbilical cord mesenchymal stem cells treated with siRNA against ELFN1-AS1 suppress colon adenocarcinoma proliferation and migration. Am J Transl Res. 2019;11(11):6989-99.

22. Lei $R$, Feng $L$, Hong D. ELFN1-AS1 accelerates the proliferation and migration of colorectal cancer via regulation of miR-4644/TRIM44 axis. Cancer Biomark. 2020;27(4):433-43.

23. Miao Z, Liu S, Xiao X, Li D. LINC00342 regulates cell proliferation, apoptosis, migration and invasion in colon adenocarcinoma via miR545-5p/MDM2 axis. Gene. 2020;743:144604.

24. Wan J, Deng D, Wang X, Wang X, Jiang S, Cui R. LINC00491 as a new molecular marker can promote the proliferation, migration and invasion of colon adenocarcinoma cells. Onco Targets Ther. 2019;12:6471-80. 
25. Dai Y, Grant S. BCL2L11/Bim as a dual-agent regulating autophagy and apoptosis in drug resistance. Autophagy. 2015;11(2):416-8.

26. Luo S, Rubinsztein DC. BCL2L11/BIM: a novel molecular link between autophagy and apoptosis. Autophagy. 2013;9(1):104-5.

27. Wood CD, Veenstra H, Khasnis S, Gunnell A, Webb HM, Shannon-Lowe C Andrews S, Osborne CS, West MJ. MYC activation and BCL2L11 silencing by a tumour virus through the large-scale reconfiguration of enhancerpromoter hubs. Elife. 2016;5:e18270.

28. Tang W, Li J, Liu H, Zhou F, Liu M. MiR-106a promotes tumor growth, migration, and invasion by targeting BCL2L11 in human endometrial adenocarcinoma. Am J Transl Res. 2017;11(9):4984-93.
29. Chen J Lin Y Jia Y XUT, WU F Jin Y LncRNA HAND2-AS1 exerts anti-oncogenic effects on ovarian cancer via restoration of $B C L 2 L 11$ as a sponge of microRNA-340-5p. J Cell Physiol. 2019;234(12):23421-36.

30. Shen Z, Chen X, Li Q, Ye H, Li J, Zhou C, Duan S. TGFB2 and BCL2L11 methylation in male laryngeal cancer patients. Oncol Lett. 2016;12(4):2999-3003.

\section{Publisher's Note}

Springer Nature remains neutral with regard to jurisdictional claims in published maps and institutional affiliations.
Ready to submit your research? Choose BMC and benefit from:

- fast, convenient online submission

- thorough peer review by experienced researchers in your field

- rapid publication on acceptance

- support for research data, including large and complex data types

- gold Open Access which fosters wider collaboration and increased citations

- maximum visibility for your research: over 100M website views per year

At BMC, research is always in progress.

Learn more biomedcentral.com/submissions 